\title{
Mechanism of local hardening in metallic glass during He ion irradiation
}

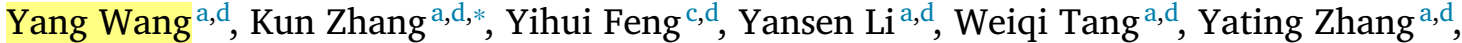 \\ Bingchen Wei ${ }^{\mathrm{a}, \mathrm{b}, \mathrm{d}, *}$, Zheng $\mathrm{Hu}^{\mathrm{e}}$ \\ ${ }^{a}$ CAS Key Laboratory of Microgravity (National Microgravity Laboratory), Institute of Mechanics, Chinese Academy of Sciences, Beijing 100190, China \\ ${ }^{\mathrm{b}}$ Center of Materials Science and Optoelectronics Engineering, University of Chinese Academy of Sciences, Beijing 100049, China \\ c State Key Laboratory of Nonlinear Mechanics, Institute of Mechanics, Chinese Academy of Sciences, Beijing 100190, China \\ ${ }^{d}$ School of Engineering Science, University of Chinese Academy of Sciences, Beijing 100049, China \\ ${ }^{\mathrm{e}}$ Science and Technology on Vehicle Transmission Laboratory, China North Vehicle Research Institute, Beijing 100072, China
}

\section{A R T I C L E I N F O}

\section{Keywords:}

Metallic glass

Ion irradiation

Local hardening

He bubble

Nanoindentation

\begin{abstract}
A B S T R A C T
When exposed to He radiation, metallic glasses (MGs) often undergo a unique transformation and mechanical evolution, unlike their crystalline counterparts. However, the relationship between He bubble evolution and the mechanical response is largely unknown. Here, a localized hardness change along ion irradiation direction was discovered under the condition that He ion irradiation does not induced nanocrystallizaiton. The location of the localized hardening peak shifted to the right, and this was accompanied by nucleation and growth of the He bubbles as the fluence increased. Besides, coalescence and overlap of the He bubbles were observed in the central of the bubble layer at the highest fluence. The localized hardness change was quantified by a model based on the He bubbles size and density. The results of this study provide insights into the effect of He bubbles on mechanical evolution and pave the way for designing MGs suitable for the nuclear industry.
\end{abstract}

\section{Introduction}

Metallic glasses (MGs) have attracted tremendous attention in the past few decades and are considered a new class of structural materials due to their attractive physical and chemical properties [1-3]. Their response to radiation is also significantly different from crystalline solids due to the lack of self-interstitials, vacancy loops, and grain boundaries [4-7]. In addition, the density of MGs is lower than that of their crystalline counterparts, providing more locations and channels for ion diffusion [8-10]. The effect of this structure on the properties of the material during irradiation, especially as it relates to the mechanical behavior of MGs, has been widely investigated [11-14]. For instance, the unlimited swelling of the crystalline solids under continuous irradiation, and the expansion or contraction of MGs following irradiation [15,16]. Irradiation generally embrittles crystalline solids, and studies have shown that some irradiated MGs become more ductile and soft and retain a fully amorphous state [17]. This occurs because radiation can induce excessive free volume in localized regions, and these "defects" can anneal out in MGs $[18,19]$. However, once nanocrystals are formed, the precipitated nanoparticles can induce the pinning of shear bands [20,21], which in turn results in reversed ductility and hardness changes. Moreover, energetic swift-heavy ion irradiation in the electronic stopping regime leads to the "ion hammer" and the viscous surface flow effect [18].

When helium ions $(\mathrm{He})$ are irradiated, in addition to the radiation effects, high-flux He implantation can lead to He ion precipitation into bubbles and voids due to the extremely low solubility and fast diffusion of $\mathrm{He}$ atoms $[15,16,19]$. In crystalline solids, dislocation loops and $\mathrm{He}$ bubbles are treated as two separate pinning centers to cause hardening in the grain interior [22,23]. The irradiation-induced hardening of conventional crystalline solids has been attributed to the strong hindrance of dislocation movement due to the generated defects and the weak obstacles resulting from the formation of He bubbles [22]. Nonetheless, it is generally considered that MGs possess no translational symmetry, therefore contains no conventional crystal defect. This lack of lattice sites leads to the potential for MGs to be suitably used as structural components in nuclear systems $[19,24]$. However, the resistance to crystallization of MGs will directly affect their suitability in these types of environments [25], He bubble evolution, as well as the nature of MG composition, such as atomic spacing [26] and the binding ability for trapped He [24]. Among this processes, a sound understanding of the He bubble evolution in MGs without crystallization is important not only from an academic point of view to understand the physical processes occurring in MGs under high doses He irradiation but also as the foundation for

\footnotetext{
* Corresponding author.

E-mail addresses: zhangkun@imech.ac.cn (K. Zhang), weibc@imech.ac.cn (B. Wei).
} 

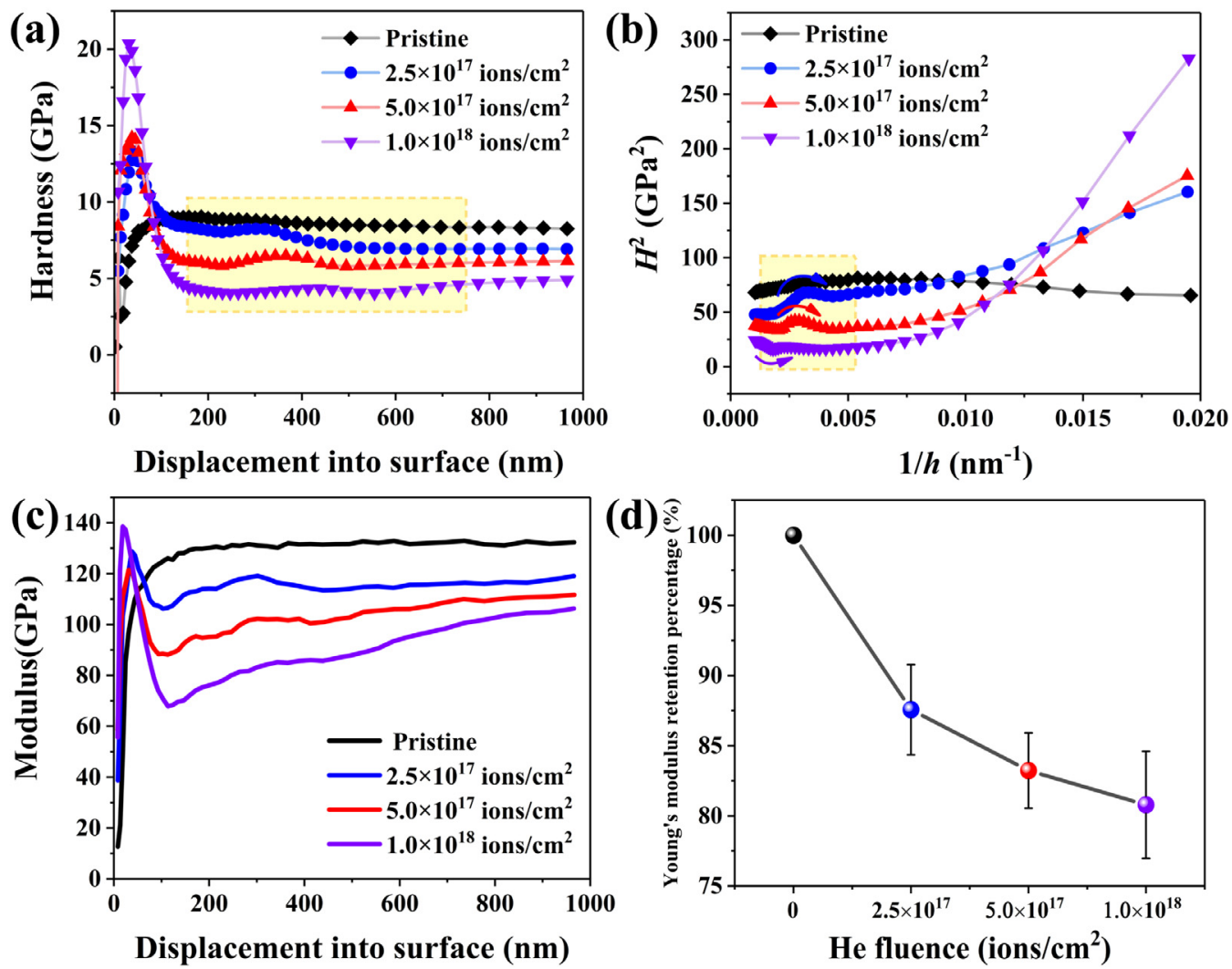

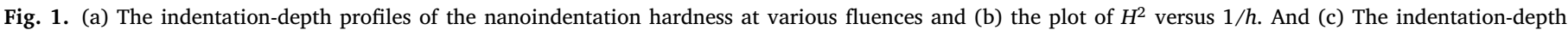

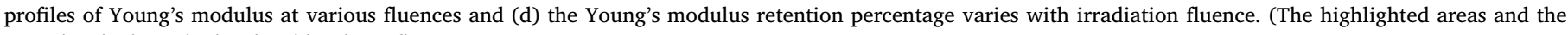
arrowheads show the localized hardness fluctuation).

developing a new class of structural materials with unprecedented radiation resistance. Although numerous studies have been conducted on the evolution and microstructure of He bubbles, the effects of He bubbles on changes in the hardness of MGs remain not clear. In the current work, ZrTiHfCuBeNi MG was chosen to investigate the effects of He bubbles on the hardness evolution during irradiation. A localized hardness fluctuation along the ion irradiation direction was observed. The kinetics and related mechanism associated with the hardness change were discussed.

\section{Materials and methods}

The bulk metallic glass ZrTiHfCuBeNi, were synthesized via a copper mould suction casting technique in a Ti-gettered high-purity argon atmosphere. Then $\Phi 5 \mathrm{~mm}$ cylindrical rods were cut into wafer samples with $2.0 \mathrm{~mm}$ thickness. Irradiation experiments were conducted at room temperature with $100 \mathrm{keV} \mathrm{He}{ }^{+}$ions using a $400 \mathrm{kV}$ electrostatic accelerator. The selected maximum ion fluence value is up to $1.0 \times 10^{18}$ ions $/ \mathrm{cm}^{2}$. The corresponding displacements per atom (dpa) at various fluences were calculated using a TRIM-code in SRIM 2008 software [23], as plotted in Fig. S1. Structural analysis of the sample was characterized by X-Ray diffraction (XRD) with $\mathrm{Cu}-\mathrm{K} \alpha$ radiation. XRD patterns of pristine and irradiated MGs both exhibit only one broad diffraction peak without any sharp Bragg peaks, further confirming the glassy structure, as shown in Fig. S2. Thermal stability and phase transformation of ZrTiHfCuBeNi MG were investigated by differential scanning calorimetry (DSC) experiments at a constant heating rate of $20 \mathrm{~K} / \mathrm{min}$. The measured glass transition temperature $\left(T_{\mathrm{g}}\right)$ and onset temperature of the crystallization $\left(T_{\mathrm{x}}\right)$ are $410{ }^{\circ} \mathrm{C}$ and $460{ }^{\circ} \mathrm{C}$, respectively, as shown in the DSC traces in Fig. S3, which are similar to the findings of previous studies $[27,28]$. Nanoindentation experiments were subsequently performed using a Nano Indenter G200 (Agilent Technologies) equipped with a Berkovich-type indentation tip in the continuous stiffness measurement (CSM) technique. Each sample was subjected to five trials and the maximum indentation depth was up to $1000 \mathrm{~nm}$. Transmission electron microscopy (TEM) observations were performed via an FEI Tecnai G2 microscope operating at $200 \mathrm{kV}$ to investigate the microstructural evolution of the samples. The cross-sectional TEM samples were obtained via the gallium focused-ion beam (FIB) lift-out technique on a ZEISS Auriga FIB-SEM workstation.

\section{Results and discussion}

Fig. 1 (a) shows the indentation-depth profiles of the nanoindentation hardness of the pristine and irradiated MGs. A considerable decrease in the nanoindentation hardness of the MGs was observed. This was attributed to the irradiation-induced increase in the liquid-like zone content, and no enhanced phase, e.g. nanocrystalline, appeared to prohibit the formation and propagation of the shear bands during irradiation [24,29]. Even more interestingly, unlike most monotonically decreasing indentation-depth curves (at the indentation depth of $h>50$ $\mathrm{nm})$ without irradiation $[30,31]$, there is a noticeable increase in the localized hardness at the low fluence of $2.5 \times 10^{17} \mathrm{ions} / \mathrm{cm}^{2}$. The hardness initially increased and then rapidly decreased at the depth of $\sim 300 \mathrm{~nm}$. Likewise, at the moderate fluence of $5.0 \times 10^{17} \mathrm{ions} / \mathrm{cm}^{2}$, the location of the peak moved to the right and the value increased. Nevertheless, when the fluence reached higher up to $1.0 \times 10^{18} \mathrm{ions} / \mathrm{cm}^{2}$, a reversal in the hardness occurred, i.e., the hardness decreased slightly at a depth of $\sim 550 \mathrm{~nm}$ and then recovered rapidly. The plot of $H_{2}$ versus $1 / h$ was created based on the Nix-Gao model, $H=H_{0} \sqrt{1+\frac{h^{*}}{h}}$, to describe this behavior in more detail (Fig. 1(b)). Here, $H$ is the hardness corresponding to the depth of indentation $h, H_{0}$ is related to the 


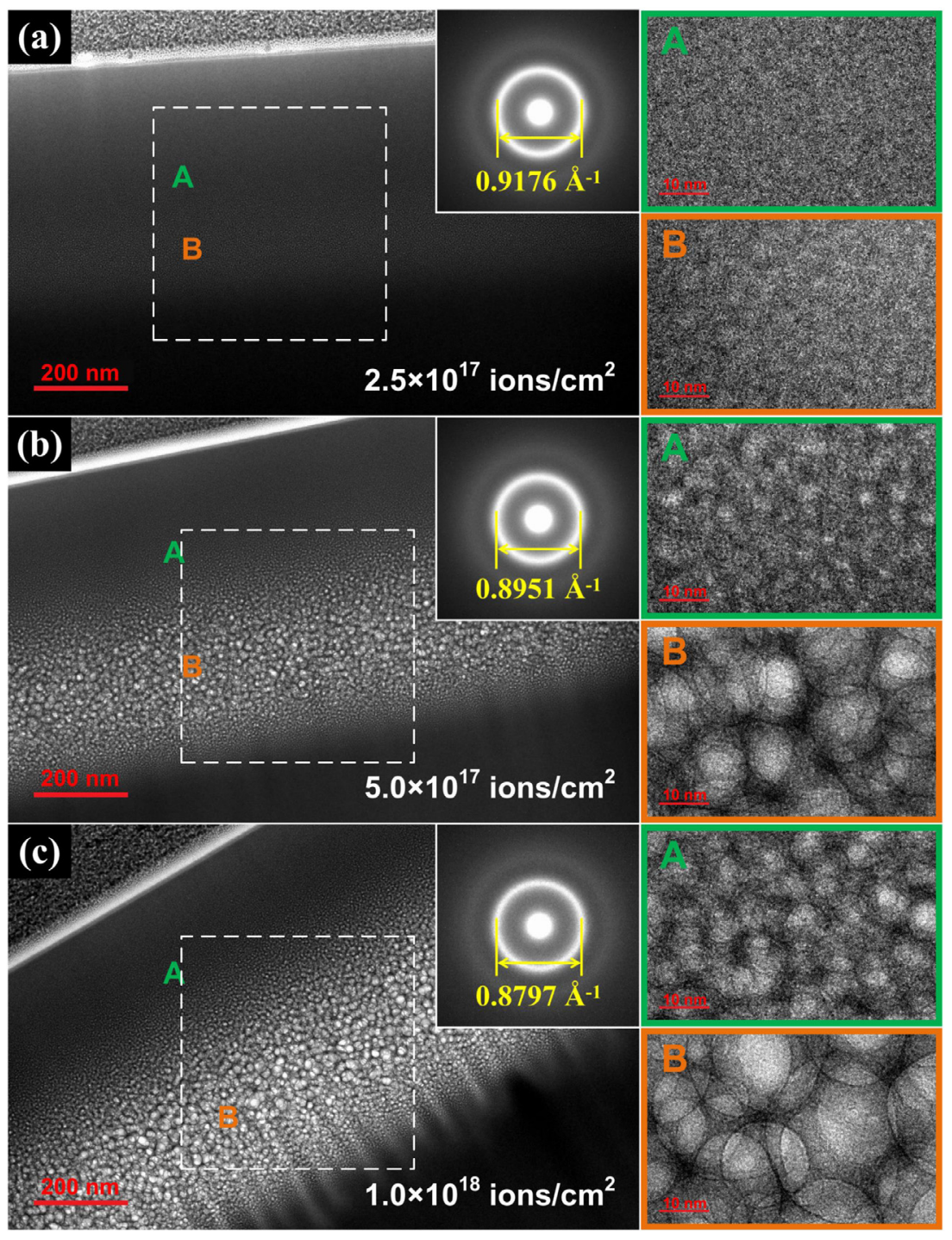

Fig. 2. Cross-sectional micrographs showing the morphology of the MGs irradiated at different dosages; (a) $2.5 \times 10^{17}$ ions $/ \mathrm{cm}^{2}$, (b) $5.0 \times 10^{17}$ ions $/ \mathrm{cm}^{2}$, and (c) $1.0 \times 10^{18}$ ions $/ \mathrm{cm}^{2}$. The left column shows the bright-field images and the insets show the SAED patterns. The right column shows different HRTEM images of the He bubbles in different regions. macroscopic hardness, and $h^{*}$ is the characteristic length. It is evident in Fig. 1(b) that there was a nonlinear localized hardness change in the fluctuation stage, i.e., a peak was observed when the fluence was below $1.0 \times 10^{18}$ ions $/ \mathrm{cm}^{2}$, whereas a valley was observed at the high fluence. In general, the stressed volume from an indent can range from 4 to 10 times the displacement into the surface in the nanoindentation test. The reason for this difference is probably the fact that a plastic zone with an approximately hemispherical shape is induced by indentation under the surface and its radius is about 4-10 times of the depth which the indenter tip reaches [32,33]. Hardness values at the depths of hardness peaks are affected by the whole plastic zone. The dpa levels and He concentrations around their peaks are much higher than other regions and defects around the peaks are much higher in density or size [34]. Therefore, we suggest the peak of local hardening in fact indicates the effects of defects around the peak displacement damage and peak He concentration regions. Moreover, Fig. 1(c) shows the indentation-depth profiles of the Young's modulus at various fluences. It is found that the modulus decreases accordingly with the increase of irradiation fluences. For comparing the modulus changes before and after irradiation, the retention percentage of Young's modulus depending on the irradiation fluence is plotted in Fig. 1(d). Here, the retention percentage is defined as the percentage ratio of the Young's modulus after implantation to the one before implantation. It can be seen that the Young's modulus retention percentage decreases as the He fluence increases. When the fluence reaches $1.0 \times 10^{18}$ ions $/ \mathrm{cm}^{2}$, the Young's modulus declines by about $20 \%$. This reduction is related to the He irradiation induced structure changes, such as the weakened atomic bonding and He bubble induced porous structures [35].

TEM observations were conducted to examine the nanoscale structure and He bubble evolution induced by the irradiation. The cross-sectional TEM images of the MGs irradiated at various fluences are presented in Fig. 2. As shown in Fig. 2(a), a band like structure corresponding to the He concentration layer is clearly visible at a depth from $400 \mathrm{~nm}$ to $600 \mathrm{~nm}$ (B region, the center of the He concentration layer), which is deeper than the He projected range. More detail, many small and irregularly voids are visible in the B region, suggesting a relatively low mass/atomic number density. These are related to the generation of He clusters. Whereas from the surface to about $300 \mathrm{~nm}$ (A region, the edge of the He concentration layer), voids are barely observed. However, at the moderate fluence, as shown in Fig. 2(b), a He bubble layer appeared at the depth of $300 \mathrm{~nm}-650 \mathrm{~nm}$ below the surface, and many well-defined He bubbles of irregular shapes were concentrated in the B region. The He bubbles from the center to bottom sides gradually decreased in size to a few nanometers. In contrast, at the highest fluence, as shown in Fig. 2(c), the He bubble layer was wider, and the He bubbles were larger. Meanwhile, the bubbles in the B region began to coalesce and even overlap, and the edges became more rounded and more distinct. The He bubble growth and overlapping 

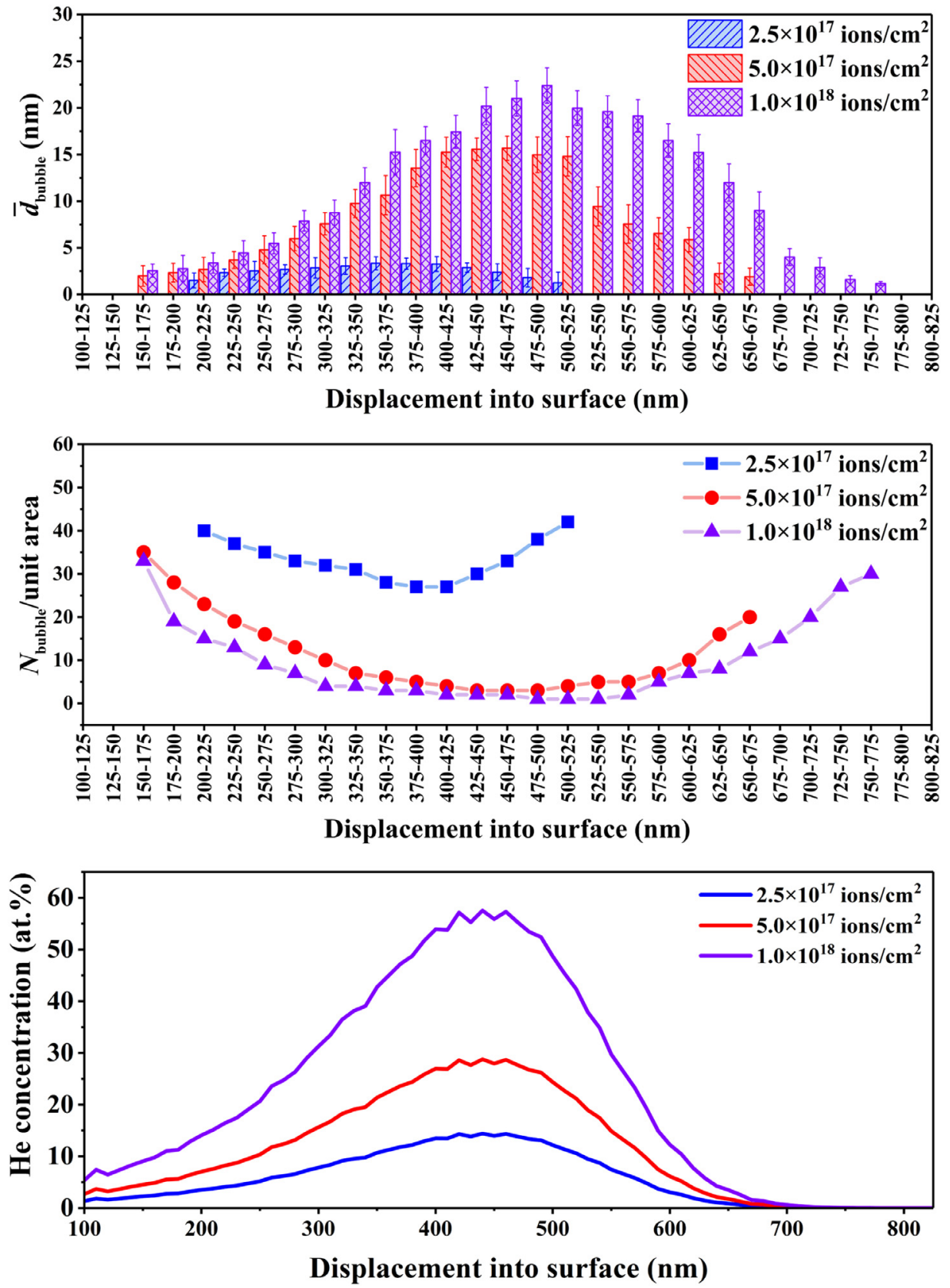

Fig. 3. Plot of the depth-dependence of (a) the average size and (b) density of He bubbles derived from extensive cross-sectional TEM analysis. (c) $\mathrm{He}$ concentration distribution calculated in the TRIMcode. process is similar to previous researches [36,37]. Moreover, as the fluence increased, the He clusters in A Region continued to accumulate and grow, eventually forming He bubbles of appreciable size at the highest fluence. If the irradiation level continuous increased, the He bubbles could not accommodate more helium to maintain a mechanical equilibrium between the internal pressure and the sintering stress [38], and then embrittlement occurred [39]. The selected-area electron diffraction (SAED) patterns were taken from the whole region including $\mathrm{He}$ bubble layers, as marked with the white dotted box in Fig. 2(a-c). Only one halo ring can be observed in both SAED patterns, indicating that the MGs maintained a fully glassy structure without any nanocrystallizaiton after irradiation. The radius of the diffraction halo decreased (from $0.91761 / \AA$ to $0.87971 / \AA$ ) with the increase in the irradiation fluence, implying an expansion of the mean nearest neighbor atomic spacing with an increase in the liquid-like zone content [28,40-42]. Xie et al. [43] have reported that the hardness enhancement had been observed in all He irradiated $\mathrm{Cu}_{50} \mathrm{Zr}_{45} \mathrm{Ti}_{5}$ glassy alloy, due to both nanocrystal formation and bubble formation. Here, He irradiation does not induced nanocrystallizaiton in ZrTiHfCuBeNi MGs, which implying that the localized hardness change may only be attributed to the bubble formation. Therefore, the bubble size and density distribution were calculated to obtain quantitative information on the evolution of the He bubbles.

Fig. 3(a) shows the average bubble size in the irradiation direction. At low fluence, the bubble size was almost evenly distributed, with an average bubble diameter of $\sim 3 \mathrm{~nm}$. While at the moderate fluence, a peak of the bubble size appeared at a depth of $\sim 470 \mathrm{~nm}$. The maximum bubble diameter is about $15 \mathrm{~nm}$. However, at the highest fluence, the peak in the bubble size shifted to the right and the maximum bubble diameter increased to more than $20 \mathrm{~nm}$. The He bubble density $N_{\text {bubble, }}$, which is the number of bubbles per unit area $(25 \mathrm{~nm} \times 25 \mathrm{~nm})$ was determined at different depths. As shown in Fig. 3(b), there was no significant difference in the He bubble density between $1.0 \times 10^{18}$ and $5.0 \times 10^{17}$ ions $/ \mathrm{cm}^{2}$, suggesting that the nucleation of the He bubbles might have reached saturation. Fig. 3(c) shows the concentration of He atoms, as 
calculated by SRIM. Notably, the peaks of the calculated He concentration were all located at depths of $\sim 450 \mathrm{~nm}$. There was an apparent difference between the peaks of the He concentration and the bubble size distribution. In fact, the SRIM-calculated depth-dependent dose level is usually determined under the implicit assumption that the target does not undergo any significant changes in volume during the process, in particular SRIM ignores the effect of injected ions and void swelling on the redistribution of the dose and injected ion profiles. However, in the present study, continuous He penetration led to the microstructural changes inside the glassy matrix, such as void swelling and He bubble induced higher porosity. These microstructural changes further complicated the injected ion and bubble profiles due to the volume averaged change in MG density causing ions to penetrate to deeper depths compared to fully dense materials [44-46].

Generally, in crystalline solids, irradiation hardening is ascribed to the interaction of irradiation-induced defects, of which two types of approximate defect hindrance models have been used. The Orowan model $[47,48]$ is used for strong hindrances (i.e., interstitials, interstitial loops, and precipitates), and the Friedel-Kroupa-Hirsch (FKH) model [49-51] is used for weak hindrances (i.e., He bubbles). In MGs, the increase in the irradiation-induced liquid-like zones cause a decrease in the global hardness. On the other hand, since He atoms have a very small atomic radius and differ greatly from alloying atoms in MGs, it is not likely that He atoms will diffuse in a way involving collective movements. Also, as a noble gas, He atoms will not stick chemically to other atoms. Their super fast diffusion and their low solubility favor bubble formation. Actually, the plastic deformation of MGs during nanoindentation is essentially different from that in crystalline solids, which is usually caused by intense shearing in narrow shear bands. The plastic flow is confined in the tiny space along the indenter tip [52,53]. Therefore, the propagating shear bands will be inhibited or slowed once they encounter a He bubble or its boundary during nanoindentation. On the other hand, ion irradiation can result in the deformation transition from a single shear band to the simultaneous operation of multiple shear bands in MG [24,40,54,55], but the formation of He bubbles may also affect the formation of multiple shear bands. As a result, the He bubble may act as the obstacle to affect the motion of the local shear bands during deformation, and eventually leading to the local hardness change. Since no crystalline phases or precipitates have been detected in the irradiated MGs, the increase in the localized hardness, $\Delta H$, is closely related to the size and density of the He bubbles. The modified localized hardness values have been plotted against $\bar{d} N_{\text {bubble }}$ values at all three irradiation fluences, as shown in Fig. 4. Interestingly, when the fluence was below $1.0 \times 10^{18}$ ions $/ \mathrm{cm}^{2}$, the calculated $\bar{d} N_{\text {bubble }}$ was in good agreement with the change in localized hardness. Here, we propose a semiquantitative bubble-induced hardening model for MGs to describe the dependence of the localized hardening on the He bubbles:

$\Delta H \propto \bar{d} N_{\text {bubble }}$

where $\bar{d}$ is the average bubble size and $N_{\text {bubble }}$ is the bubble density. In addition, according to early studies of He bubble [56-59], elastic interactions between two pressurized bubbles can lead to an attractive force between them. As He bubbles grow rapidly, the attractive force between them increases, which increases the internal stress inside the glassy matrix and may lead to an increase in the local hardening in the areas of He concentration [60]. However, at the highest fluence, the change in localized hardness was not correlated with the calculated $\bar{d} N_{\text {bubble }}$. In my opinion, as the bubbles grow and exceed a critical size, the localized liquid-like zone near the He bubbles do not provide sufficient space for their continued growth. The overlapping bubbles may have a negative effect on the localized hardening of the MGs. Moreover, the continuous coalescence and overlapping of the He bubbles may have resulted in large voids or open areas in the bubble layer, which eventually result in softening because of the collapse of the glassy structure [23].

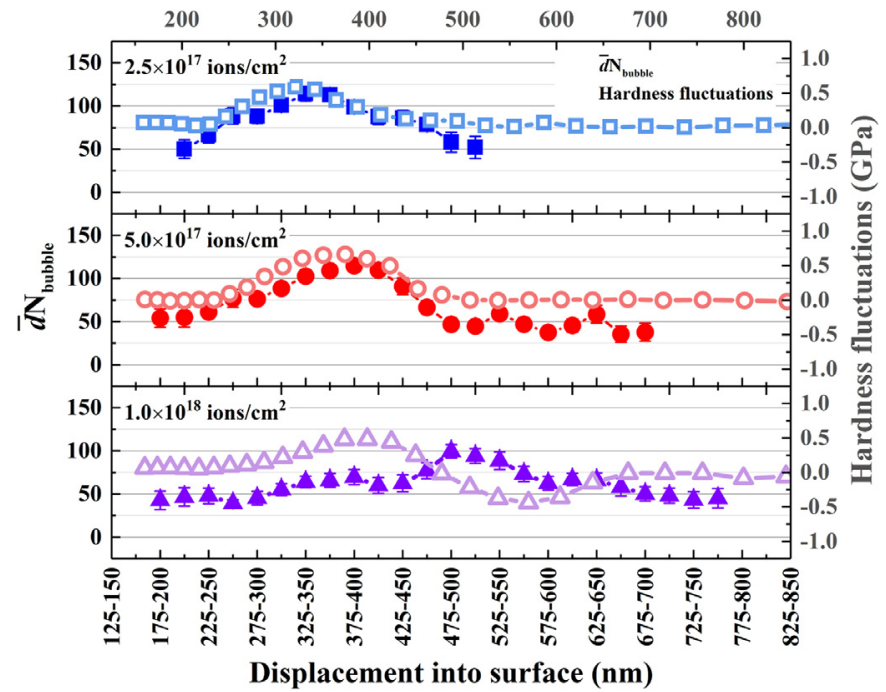

Fig. 4. Calculated $\bar{d} N_{\text {bubble }}$ as a function of depth and the local hardening for $\mathrm{He}^{+}$irradiated MGs at various fluences.

\section{Conclusions}

The microstructure evolution and mechanical properties of ZrTiHfCuBeNi MGs under He irradiation were investigated. A localized hardness change along ion irradiation direction was discovered under the condition that He ion irradiation does not induced nanocrystallizaiton. The peak position of the localized hardness shifted to the right as the irradiation fluence increased, and this is accompanied by the nucleation and growth of the He bubbles. In addition, coalescence and overlap of the He bubbles were observed in the central region of the bubble layer. The change in the localized hardness is well described by a semiquantitative model based on the size and density of the He bubbles. However, at high fluence, the overlapping bubbles might have a negative role on the localized hardening in the MGs. The results of this study provide insights into the effects of He bubbles on the hardness evolution in MGs.

\section{Declaration of Competing Interests}

The authors declare that they have no known competing financial interests or personal relationships that could have appeared to influence the work reported in this paper.

\section{Acknowledgments}

This work was supported by the National Natural Science Foundation of China (Grant No. 51401028, No. 51271193, No. 11402277, No. 11790292) and the Strategic Priority Research Program of the Chinese Academy of Sciences (Grant No. XDB22040303). This work was also supported by the Innovation Program (237099000000170004).

\section{References}

[1] J. Luo J, Y. Shi, Acta Mater. 82 (2015) 483-490.

[2] H.F. Zhou, C. Zhongac, Q.P. Cao, S.X. Qu, X.D. Wang, W. Yang, J.Z. Jiang, Acta Mater. 68 (2014) 32-41.

[3] W.H. Wang, Prog. Mater Sci. 57 (2012) 487-656.

[4] J. Antonaglia, W.J. Wright, X. Gu, R.R. Byer, T.C. Hufnagel, M. LeBlanc, J.T. Uhl, K.A. Dahmen, Phys, Rev, Lett. 112 (2014) 155501.

[5] L. Tian, Y.Q. Cheng, Z.W. Shan, J. Li, C.C. Wang, X.D. Han, J. Sun, E. Ma, Nat. Commun. 3 (2012) 609.

[6] O.V. Kuzmin, Y.T. Pei, C.Q. Chen, J.T.M. De Hosson, Acta Mater. 60 (2012) 889-898. [7] K. Zhang, Z. Hu, F.J. Li, B.C. Wei, Appl. Surf. Sci. 390 (2016) 941-945.

[8] Y. Huang, B. Zhou, H. Fan, Y. Wang, D. Wang, J. Sun, J. Shen, Mater. Design (1980-2015) 62 (2014) 133-136.

[9] J. Carter, E.G. Fu, M. Martin, G. Xie, X. Zhang, Y.Q. Wang, D. Wijesundera, X.M. Wang, W.-K. Chu, L. Shao, Scr. Mater. 61 (2009) 265-268. 
[10] Y. Huang, H. Fan, X. Zhou, P. Xue, Z. Ning, D. Daisenberger, J. Sun, J. Shen, Scr. Mater. 103 (2015) 41-44.

[11] W.D. Luo, B. Yang, G.L. Chen, Scr. Mater. 64 (2011) 625-628.

[12] X.N. Zhang, X.X. Mei, Q. Zhang, X.N. Li, Y.M. Wang, Y.N. Wang, Nucl. Instrum. Methods Phys. Res. Sect. B 406 (2017) 548-554.

[13] B. Wang, X. Mei, H. Zhang, W. Hou, Y. Wang, Z. Wang, C. Dong, J. Nucl. Mater. 444 (2014) 342-348.

[14] X. Zhang, X. Mei, Q. Zhang, X. Li, J. Qiang, Y. Wang, J. Nucl. Mater. 490 (2017) $216-225$.

[15] H.F. Huang, W. Zhang, M. De Los Reyes, X.L. Zhou, C. Yang, R. Xie, X.T. Zhou, P. Huai, H.J. Xu, Mater. Design 90 (2016) 359-363.

[16] K. Zhang, Z. Hu, Z. Zhao, B. Wei, Y. Li, Y. Wei, Appl. Surf. Sci. 437 (2018) 176-180.

[17] J. Brechtl, H. Wang, N.A.P.K. Kumar, T. Yang, Y.-R. Lin, H. Bei, J. Neuefeind, W. Dmowski, S.J. Zinkle, J. Nucl. Mater. 526 (2019) 151771.

[18] J. Carter, E.G. Fu, G. Bassiri, B.M. Dvorak, N. David Theodore, G. Xie, D.A. Lucca, M. Martin, M. Hollander, X. Zhang, L. Shao, Nucl. Instrum. Methods Phys. Res. Sect. B 267 (2009) 1518-1521.

[19] X. Zhang, X. Mei, Q. Zhang, X. Li, J. Qiang, Y. Wang, J. Nucl. Mater. 490 (2017) $216-225$.

[20] M. Cui, J. Wang, Z. Wang, T. Shen, K. Wei, C. Yao, J. Sun, N. Gao, Y. Zhu, L. Pang, D. Wang, H. Zhu, Y. Han, X. Fang, Nucl. Instrum. Methods Phys. Res. Sect. B 406 (2017) 611-617.

[21] J. Brechtl, S. Agarwal, M.L. Crespillo, J. Salasin, T. Yang, H. Bei, S.J. Zinkle, Intermetallics 116 (2020) 106655

[22] M. Callisti, M. Karlik, T. Polcar, J. Nucl. Mater. 473 (2016) 18-27.

[23] Y. Wang, K. Zhang, Y. Feng, Y. Li, W. Tang, B. Wei, Entropy 20 (2018) 835.

[24] Y. Wang, K. Zhang, Y.H. Feng, Y.S. Li, W.Q. Tang, Y.T. Zhang, B.C. Wei, Z. Hu, J. Nucl. Mater. 527 (2019) 151785.

[25] M.I. qbal, J.I. Akhter, Z.Q. Hu, H.F. Zhang, A. Qayyum, W.S. Sun, J. Non Cryst. Solids 353 (2007) 2452-2458.

[26] X. Tong, G. Wang, Z.H. Stachurski, J. Bednarčík, N. Mattern, Q.J. Zhai, J. Eckert, Sci. Rep. 6 (2016) 1-12.

[27] Y. Tong, J.C. Qiao, C. Zhang, J.M. Pelletier, Y. Yao, J. Non Cryst. Solids 452 (2016) $57-61$.

[28] P. Gong, K.F. Yao, H.Y. Ding, Mater. Lett. 156 (2015) 146-149.

[29] X.L. Bian, G. Wang, H.C. Chen, L. Yan, J.G. Wang, Q. Wang, P.F. Hu, J.L. Ren, K.C. Chan, N. Zheng, A. Teresiak, Y.L. Gao, Q.J. Zhai, J. Eckert, J. Beadsworth, K.A. Dahmen, P.K. Liaw, Acta Mater. 106 (2016) 66-77.

[30] W.D. Nix, H. Gao, J. Mech. Phys. Solids 46 (1998) 411-425.

[31] T. Miyazawa, T. Nagasaka, R. Kasada, Y. Hishinuma, T. Muroga, H. Watanabe, T. Yamamoto, S. Nogami, M. Hatakeyama, J. Nucl. Mater. 455 (2014) 440-444.

[32] H. Tanigawa, S. Jitsukawa, A. Hishinuma, M. Ando, Y. Katoh, A. Kohyama, T. Iwai, J. Nucl. Mater. 283 (2000) 470-473.

[33] H.-H. Jin, C. Shin, D.H. Kim, K.H. Oh, J.H. Kwon, Nucl. Instrum. Methods Phys. Res. Sect. B 266 (2008) 4845-4848.
[34] M.H. Cui, J. Wang, Z.G. Wang, T.L. Shen, K.F. Wei, C.F. Yao, J.R. Sun, N. Gao, Y.B. Zhu, L.L. Pang, D. Wang, H.P. Zhu, Y. Han, X.S. Fang, Nucl. Instrum. Methods Phys. Res. Sect. B 406 (2017) 611-617.

[35] F. Zhu, D. Wang, N. Gao, H.Q. Peng, Z. Xie, Z.J. Zhang, J. Nucl. Mater. 518 (2019) 226-233.

[36] T.Y. Wang, H. Kim, J.G. Gigax, J.Y. Fan, K.L. Peddicord, E. Fu, A. Zare, D.A. Lucca, L. Shao, Nucl. Instrum. Methods Phys. Res. Sect. B 451 (2019) 1-5.

[37] L. Shao, B.P. Gorman, A. Aitkaliyeva, N.D. Theodore, G.Q. Xie, Appl. Phys. Lett. 101 (2012) 041901.

[38] S.B. Gilliam, S.M. Gidcumb, N.R. Parikh, D.G. Forsythe, B.K. Patnaik, J.D. Hunn, L.L. Snead, G.P. Lamaze, J. Nucl. Mater. 347 (2005) 289-297.

[39] R. Liontas, X.W. Gu, E. Fu, Y. Wang, N. Li, N. Mara, J.R. Greer, Nano Lett. 14 (2014) 5176-5183.

[40] H. Chen, Y. Hai, R. Liu, Q. Lei, L. Ye, J. Xu, G. Wang, W. Yin, L. Yan, X. Zhou, Intermetallics 104 (2019) 52-58.

[41] S. Michalik, J. Michalikova, M. Pavlovic, P. Sovak, H.-P. Liermann, M. Miglierini, Acta Mater. 80 (2014) 309-316.

[42] X. Xiao, Q. Chen, H. Yang, H. Duan, J. Qu, J. Nucl. Mater. 485 (2017) 80-89.

[43] G.Q. Xie, L Shao, D.V. Louzguine-Luzgina, A. Inoue, Surf. Coat. Technol. 206 (2011) 829-833

[44] J. Wang, M.B. Toloczko, N. Bailey, F.A. Garner, J. Gigax, L. Shao, Nucl. Instrum. Methods Phys. Res. Sect. B 387 (2016) 20-28.

[45] W.G. Johnston, J.H. Rosolowski, A.M. Turkalo, T. Lauritzen, J. Nucl. Mater. 47 (1973) 155-167.

[46] G.R. Odette, D.M. Schwartz, A.J. Ardell, Radiat. Eff. 22 (1974) 217-223.

[47] L. Yu, X. Xiao, L. Chen, H. Chu, H. Duan, Int. J. Solids Struct. 102-103 (2016) 267-274

[48] J. Dérès, L. Proville, M.-C. Marinica, Acta Mater. 99 (2015) 99-105.

[49] S.J. Zinkle, Y. Matsukawa, J. Nucl. Mater. 329-333 (2004) 88-96.

[50] F. Kroupa, P.B. Hirsch, Discuss. Faraday Soc. 38 (1964) 49-55.

[51] N. Li, E.G. Fu, H. Wang, J.J. Carter, L. Shao, S.A. Maloy, A. Misra, X. Zhang, J. Nucl. Mater. 389 (2009) 233-238.

[52] A.L. Greer, Y.Q. Cheng, E. Ma, Mater. Sci. Eng. 74 (2013) 71-132.

[53] Y.S. Li, K. Zhang, Y. Wang, W.Q. Tang, Y.T. Zhang, B.C. Wei, Z. Hu, Mater. Sci. Eng. 773 (2020) 138844

[54] C.A. Schuh, T.G. Nieh, Acta Mater. 51 (2003) 87-99.

[55] Y.I. Golovin, V.I. Ivolgin, V.A. Khonik, K. Kitagawa, A.I. Tyurin, Scr. Mater. 45 (2001) 947-952.

[56] J.D. Eshelby, Ann. Phys. Lpz. 456 (1957) 116-121.

[57] A.B. Lidiard, R.S. Nelson, Philos. Mag. 17 (1968) 425-429.

[58] J.R. Willis, R. Bullough, J. Nucl. Mater. 32 (1969) 76-87.

[59] F. Chalon, F. Montheillet, J. Appl. Mech. 70 (2003) 789-798.

[60] L. Shao, B.P. Gorman, A. Aitkaliyeva, N.D. Theodore, G.Q. Xie, Appl. Phys. Lett. 101 (2012) 041901. 